\title{
A LATTICE CONSTRUCTION AND CONGRUENCE-PRESERVING EXTENSIONS
}

\author{
G. GRÄTZER AND E. T. SCHMIDT
}

\begin{abstract}
A chopped lattice is a partial lattice we obtain from a bounded lattice by removing the unit element.

Under a very natural condition, (FG), the finitely generated ideals of a chopped lattice $M$ form a lattice which is a congruence-preserving extension of $M$; that is, every congruence of $M$ has exactly one extension to this lattice.

In this paper, we investigate how we can obtain from a pair of lattices $A$ and $B$ by amalgamation a chopped lattice. We establish a set of six sufficient conditions.

We then investigate when the chopped lattice so obtained will satisfy Condition (FG). A typical result is the following: if $C=A \cap B$ is a principal ideal of both $A$ and $B$ and $A$ is modular, then Condition (FG) holds.

We apply this to prove that if $L$ is a lattice with a nontrivial distributive interval, then $L$ has a proper congruence-preserving extension.
\end{abstract}

\section{INTRODUCTION}

To find a simple proof of the congruence lattice characterization theorem of finite lattices, H. Lakser and the first author (see [1]) introduced a special type of finite partial lattices: a meet-semilattice in which any two elements with a common upper bound have a join. If $M$ is such a finite partial lattice, then the ideal lattice of $M$ is a congruence-preserving extension of $M$; that is, every congruence of $M$ has exactly one extension to the ideal lattice.

In [2], we introduced the name chopped lattice for such partial lattices, no longer necessarily finite. Of course, if $M$ is no longer finite, we cannot expect the ideal lattice to be a congruence-preserving extension. It is natural to consider, instead, finitely generated ideals; unfortunately, they do not, in general, form a lattice. In Section 2 we introduce Condition (FG) under which the finitely generated ideals form a lattice.

Given two lattices $A$ and $B$, sharing the sublattice $C=A \cap B$, we obtain the lattice $M(A, B)$ by amalgamation. If $C$ is a principal ideal of both $A$ and $B$, then $M(A, B)$ is a chopped lattice.

In Section 3, we introduce (see Definition 3) a set of sufficient conditions under which $M(A, B)$ is a chopped lattice. If $A$ and $B$ satisfy the conditions of Definition 3, we shall call $A, B$ a chopped pair. Theorem 1 states that if $A, B$ is a chopped pair, then $M(A, B)$ is a chopped lattice. The concept of a chopped pair does not

Date: April 10, 1993.

1991 Mathematics Subject Classification. Primary 06B10; Secondary 06B05.

The research of the first author was supported by the NSERC of Canada.

The research of the second author was supported by the Hungarian National Foundation for Scientific Research, under Grant No. 1903. 
seem strong enough to compute with it. In Section 4, we introduce two stronger versions: sharp and full chopped pairs.

In Section 5 we investigate finitely generated ideals in $M(A, B)$ for a chopped pair $A, B$. For a sharp chopped pair $A$ and $B$, if $C=A \cap B$ satisfies the Ascending Chain Condition, then we obtain Condition (FG) (which guarantees that the finitely generated ideals form a lattice) for $M(A, B)$.

In Section 6 we investigate modular lattices. If $A, B$ is a sharp chopped pair and both $A$ and $B$ are modular, then $M(A, B)$ satisfies Condition (FG) (Theorem 3). If $A, B$ is a full chopped pair, then it is enough to assume that one of them is modular to obtain the same conclusion (Theorem 4).

In Section 7 we deal with the problem whether every lattice has a proper congruence-preserving extension. We apply Theorem 4 to prove that if there exist a nontrivial distributive interval in a lattice, then it has a proper congruence-preserving extension.

A modular example of a congruence-preserving extension is outlined in Section 6.

1.1. Notation. We refer the reader to [1] for the basic concepts and notation.

In a lattice $L,[x, y]_{L}$ denotes the interval in $L$, and $(a]_{L}$ the principal ideal generated by $a$. If there is no confusion, the subscript is dropped.

If $L$ is a sublattice of $K$, then we call $K$ an extension of $L$. If $L$ has a zero, and it is also the zero of $K$, then $K$ is $\{0\}$-extension of $L$.

\section{Chopped lattices}

A chopped lattice $M$ is a lattice $L$ with zero, 0 , and unit, 1 , with the unit removed: $M=L-\{1\}$; on $M, 0$ is a nullary operation, $\wedge$ is an operation, and $\vee$ is a partial operation. Equivalently, a chopped lattice $M$ is a meet-semilattice with zero, 0 , in which any two elements having an upper bound have a join. $M$ will be regarded as a partial algebra $\langle M ; \wedge, \vee, 0\rangle$.

We shall use the concept of extension for chopped lattices; observe that, by definition, an extension of a chopped lattice is a $\{0\}$-extension.

An ideal $I$ of $M$ is a subset of $M$ containing 0 with the following two properties for $x, y \in M$ :

$x \in I$ and $y \leq x$ imply that $x \in I$.

If $x, y \in I$ and $x \vee y$ exists, then $x \vee y \in I$.

For $H \subseteq M$, there is a smallest ideal $(H]$ of $M$ containing $H$. If an ideal $I$ can be generated in the form $(H]$ for some finite set $H$, then the ideal $I$ is called finitely generated. In particular, for $a \in M$, we let $(a]=(\{a\}]$ be the principal ideal generated by $a$ in $M$, that is,

$$
(a]=\{x \mid x \in M \text { and } x \leq a\} .
$$

Id $M$ denotes the lattice of ideals of $M$. Obviously, $\operatorname{Id} M$ is a lattice. $\operatorname{Id}_{\mathrm{fg}} M$, the finitely generated ideals of $M$, form a join-sublattice of Id $M$.

By identifying $a \in M$ with $(a)$, we regard Id $M$ an extension of $M$.

Definition 1. A chopped lattice $M$ satisfies Condition (FG) if every finitely generated ideal is a finite union of principal ideals.

If $M$ satisfies Condition (FG), then $\operatorname{Id}_{\mathrm{fg}} M$ is a sublattice of $\operatorname{Id} M$. Indeed, if

$$
\begin{aligned}
& I=\left(a_{1}\right] \cup \ldots \cup\left(a_{n}\right], \\
& J=\left(b_{1}\right] \cup \ldots \cup\left(b_{m}\right],
\end{aligned}
$$


then

$$
I \cap J=\bigcup\left(\left(a_{i} \wedge b_{j}\right] \mid 1 \leq i \leq n, 1 \leq j \leq m\right) .
$$

Lemma II.3.19 in [1] states the following:

Lemma 1. Let $M$ be a finite chopped lattice. Then $\operatorname{Id} M$ is a congruence-preserving extension of $M$.

The proof of this lemma implicitly contains the following two lemmas.

Lemma 2. Let $M$ be a chopped lattice. Then every congruence relation of $M$ has an extension to Id $M$.

Proof. Let $\Theta$ be a congruence of $M$; define a relation $\bar{\Theta}$ on Id $M$ as follows:

$$
I \equiv J
$$

if for every $i \in I$ there exists a $j \in J$ such that $i \equiv j(\Theta)$, and symmetrically. The proof is the same as in [1].

Lemma 3. Let $M$ be a chopped lattice, and let $S \supseteq M$ be a sublattice of $\operatorname{Id} M$. Let us assume that in $S$ every ideal $I \in S$ is a finite union of principal ideals. Then every congruence relation of $M$ has a unique extension to $S$.

Proof. First observe that if $a \in M$ and $I \in S$, then $(a] \cap I$ is principal. Indeed,

$$
I=\left(a_{1}\right] \cup \ldots \cup\left(a_{n}\right],
$$

and so $(a] \cap I$ is generated by $\left\{a \wedge a_{1}, \ldots, a \wedge a_{n}\right\}$. Since this set has an upper bound (namely $a$ ), it has a join $b$ (since $M$ is a chopped lattice), and $b$ obviously generates $(a] \cap I$.

Let $\Phi$ be an extension of $\Theta$ from $M$ to $S$. Let $I, J \in S, I \equiv J(\Phi)$, and $a \in I$. Then $I \wedge(a] \equiv J \wedge(a](\Phi)$. By the statement in the previous paragraph, there is a $b \in J$ such that $(a] \wedge J=(b]$; obviously, $a \equiv b(\Theta)$. We conclude that $I \equiv J(\bar{\Theta})$. So $\Phi \subseteq \bar{\Theta}$.

Conversely, let $I, J \in S$ with $I \equiv J(\bar{\Theta})$. By the assumption on $S$, we can represent these ideals as

$$
\begin{aligned}
& I=\left(a_{1}\right] \cup \ldots \cup\left(a_{n}\right], \\
& J=\left(b_{1}\right] \cup \ldots \cup\left(b_{m}\right] .
\end{aligned}
$$

By the definition of $\bar{\Theta}$, for every $a_{i}$ there is a $c_{i}$ in $J$ with $a_{i} \equiv c_{i}(\Theta)$. Symmetrically, for every $b_{j}$ there is a $d_{j}$ in $I$ with $d_{j} \equiv b_{j}(\Theta)$. Since $\Phi$ is an extension of $\Theta$, these congruences hold for $\Phi$. The join of these $n+m$ congruences yields $I \equiv J$ $(\Phi)$, proving that $\bar{\Theta} \subseteq \Phi$. Thus $\bar{\Theta}=\Phi$, and so every congruence of $M$ has a unique extension to $S$.

Therefore, the following is true:

Lemma 4. Let $M$ be a chopped lattice satisfying Condition (FG). Then $\operatorname{Id}_{\mathrm{fg}} M$ is a congruence-preserving extension of $M$.

In fact, a congruence-preserving $\{0\}$-extension. 


\section{CHOPPED PAIRS}

Let $A$ and $B$ be lattice, let $C=A \cap B \neq \varnothing$. Then we can form the amalgamation $M=M(A, B)$ of $A$ and $B$ over $C$. It is well-known that on $M$ we can define a partial ordering:

Definition 2. The partial ordering $\leq_{M}$ is defined on $M$ as follows:

1. For $x, y \in A$, let $x \leq_{M} y$ iff $x \leq_{A} y$.

2. For $x, y \in B$, let $x \leq_{M} y$ iff $x \leq_{B} y$.

3. For $x \in A$ and $y \in B$, let $x \leq_{M} y$ iff there exists a $c \in C$ such that $x \leq_{A} c$ and $c \leq_{B} y$; and symmetrically, for $x \in B$ and $y \in A$.

The subscripts of $\leq$ will be dropped whenever there is no danger of confusion.

We shall use the following notation: $M(A, B)=A \cup B$ is the poset obtained by amalgamating $A$ and $B$ over $C$. In $A$ we form the ideal $I_{A}$ generated by $C$; we set $C_{A}=I_{A}-C$; symmetrically, we define $I_{B}$ and $C_{B}$. Note that the ideal $C_{M}$ generated by $C$ in $M$ is the disjoint union of $C, C_{A}$, and $C_{B}$.

Sometimes, the poset $M(A, B)$ is a chopped lattice. The next definition formulates some natural conditions under which this is the case.

Definition 3. A pair of lattices $A$ and $B$ is called a chopped pair iff the following conditions are satisfied:

1. The lattices $A$ and $B$ have a common zero 0.

2. Let $C$ denote the lattice $A \cap B$. Then $C$ has a largest element $i$.

3. For $x \in C_{M}$, there is a smallest $\bar{x} \in C$ satisfying $x \leq \bar{x}$.

4. For $x \in M(A, B)$, there is a largest $\underline{x} \in C$ satisfying $\underline{x} \leq x$.

5. For $x \in C_{A}$ and $y \in C_{B}$, the two elements: $x \vee \bar{y}$ (formed in $A$ ) and $\bar{x} \vee y$ (formed in $B$ ) are comparable (in $M(A, B)$ ).

6. For $x \in A-B$ and $y \in B-A$, the two elements: $x \wedge \underline{y}$ (formed in $A$ ) and $\underline{x} \wedge y$ (formed in $B$ ) are comparable (in $M(A, B)$ ).

Theorem 1. Let $A, B$ be a chopped pair. Then $M(A, B)$ is a chopped lattice.

Proof. There are two claims to verify.

Claim 1. $M(A, B)$ is a meet-semilattice.

Let $x, y \in M(A, B)$. We have to find $u=\inf _{M(A, B)}\{x, y\}$. We shall distinguish several cases.

Case 1.1. $x, y \in A$. Let $u=x \wedge y$ be formed in $A$. Obviously in $M(A, B), u \leq x$ and $u \leq y$. Now let $v \in M(A, B)$ be a common lower bound of $x$ and $y$. There are two subcases to consider.

Case 1.1a. $v \in A$. By Definition 2.1, $v$ is a common lower bound of $x$ and $y$ in $A$, hence, $v \leq u$.

Case 1.1b. $v \in B$. By Definition 2.3, there are elements $c_{x}$ and $c_{y}$ in $C$ such that $v \leq_{B} c_{x} \leq_{A} x$ and $v \leq_{B} c_{y} \leq_{A} y$. Then $c_{x} \wedge c_{y} \in C$, and $v \leq_{B} c_{x} \wedge c_{y} \leq_{A} u$. So indeed, $u=\inf _{M(A, B)}\{x, y\}$.

Case 1.2. $x, y \in B$. Proceed as in Case 1.1.

Case 1.3. $x \in A, y \in B$. In view of the previous cases, we can assume that $x \in A-B$ and $y \in B-A$. Since by Definition 2.3, any common lower bound must be in $C_{M}$, we can replace $x$ by $x \wedge i$ and $y$ by $y \wedge i$. So again referring to the previous cases, we can assume that $x \in C_{A}$ and $y \in C_{B}$. Now take a common lower bound $v$ of $x$ and $y$. 
Now we claim that of the common lower bounds $v \in A$, there is a largest one, $x \wedge y$. Indeed, $x \wedge y$ is a lower bound. If $t \in A$ is also a lower bound, then $t \leq y$ in $\bar{M}(A, B)$, hence by Definition 2.3 , there is a $c \in C$ satisfying $t \leq_{A} c \leq{ }_{B} y$. Obviously, $c \leq \underline{y}$, and so $t \leq_{A} x \wedge \underline{y}$, as claimed.

Now we claim that of the common lower bounds $v \in B$, there is a largest one, $\underline{x} \wedge y$. To prove this, proceed as in the previous paragraph.

Finally, by Definition 3.6, $x \wedge \underline{y}$ and $\underline{x} \wedge y$ are comparable, hence $\inf _{M(A, B)}\{x, y\}$ exists and it equals $\sup \{x \wedge \underline{y}, \underline{x} \wedge y\}$.

Case 1.4. $x \in B, y \in A$. Proceed as in Case 1.3.

This completes the proof of Claim 1.

Claim 2. In $M(A, B)$, any two elements, $x$ and $y$, having a common upper bound, $v$, have a join.

Let $x, y \in M(A, B)$, and let $v$ be an upper bound of $x$ and $y$. We have to find $u=\sup _{M(A, B)}\{x, y\}$. We shall distinguish several cases.

Case 2.1. $x, y \in A$. Form $u=x \vee y$ in $A$. We have to show that if $t$ is any upper bound of $x$ and $y$ in $M(A, B)$, then $u \leq t$.

Case 2.1a. $t \in A$. This case is obvious.

Case 2.1b. $t \in B$. By Definition 2.3, there are $c_{x}, c_{y} \in C$ so that $x \leq_{A} c_{x} \leq_{B} t$ and $y \leq_{A} c_{y} \leq_{B} t$. Therefore, $u=x \vee y \leq_{A} c_{x} \vee c_{y} \leq_{B} t$; so again, by Definition 2.3, $u \leq_{M(A, B)} t$, completing Case 2.1.

Case 2.2. $x, y \in B$. Proceed as in Case 2.1.

Case 2.3. $x \in A$ and $y \in B$. In view of Cases 2.1-2.2, we can assume that $x \in A-B$ and $y \in B-A$. Without loss of generality, we can assume that $t \in A$. It follows that $y \in C_{B}$. Again, we distinguish two subcases.

Case 2.3a. $x \in C_{A}$. If $t \in A$ is an upper bound of $x$ and $y$, then $x \vee \bar{y} \leq t$. Similarly, if $t \in B$ is an upper bound of $x$ and $y$, then $\bar{x} \vee y \leq t$. By Definition 3.5, the elements $x \vee \bar{y}$ and $\bar{x} \vee y$ are comparable, hence,

$$
\sup \{x, y\}=\inf \{x \vee \bar{y}, \bar{x} \vee y\}
$$

Case 2.3b. $x \notin C_{A}$. In this case, no upper bound of $x$ is in $B$, hence, $\sup \{x, y\}=x \vee \bar{y}$ formed in $A$.

Case 2.4. $x \in B$ and $y \in A$. Proceed as in Case 2.3.

This completes the proof of Claim 2 and of the lemma.

\section{Some examples AND SPECiAl CASES}

It is easy to give examples that last two strange conditions of Definition 3 do not follow from the others. Here is one: let $A=B$ be the direct product of the two element chain $\{0,1\}$ with the three element chain $\{0, a, 1\}$. The elements are of the form $\langle x, y\rangle$, where $x \in\{0,1\}$ and $y \in\{0, a, 1\}$. We make $A$ and $B$ disjoint (we shall denote $\langle x, y\rangle \in A$ by $\langle x, y\rangle_{A}$, and the same for $B$ ), then we identify elements as follows:

$\langle 0,0\rangle_{A}$ with $\langle 0,0\rangle_{B}$

$\langle 1,0\rangle_{A}$ with $\langle 0,1\rangle_{B}$

$\langle 0,1\rangle_{A}$ with $\langle 1,0\rangle_{B}$

$\langle 1,1\rangle_{A}$ with $\langle 1,1\rangle_{B}$

So $C=\left\{\langle 0,0\rangle_{A},\langle 1,0\rangle_{A},\langle 0,1\rangle_{A},\langle 1,1\rangle_{A}\right\}$ is a four-element Boolean lattice. It is easy to see that Definitions 3.1-3.4 hold, but both Definitions 3.5 and 3.6 fail. Indeed, let $x=\langle a, 0\rangle_{A} \in C_{A}$ and $y=\langle a, 0\rangle_{B} \in C_{B}$. Then $\bar{x}=\langle 1,0\rangle_{A}$ and 
$\bar{y}=\langle 1,0\rangle_{B}=\langle 0,1\rangle_{B}$. Hence,

$$
x \vee \bar{y}=\langle a, 1\rangle_{A} \text { and } \bar{x} \vee y=\langle a, 1\rangle_{B},
$$

and these two elements are not comparable.

If $A, B$ is a chopped pairs, then we know that in $M(A, B)$ any pair of elements with a common upper bound has a join. To perform computations we need more; we must have a formula for the join we can work with.

Definition 4. A chopped pair of lattices $A$ and $B$, is called sharp iff

$$
x \vee \bar{y}=\bar{x} \vee y,
$$

for $x \in C_{A}$ and $y \in C_{B}$, and

$$
x \wedge \underline{y}=\underline{x} \wedge y,
$$

for $x \in A-B$ and $y \in B-A$.

There are many equivalent forms of these conditions; for instance, the first is equivalent to

$$
x \vee \bar{y} \in C,
$$

for $x \in C_{A}$ and $y \in C_{B}$; or to

$$
x \vee y=\bar{x} \vee \bar{y} .
$$

Observe that if $A$ and $B$ form a sharp chopped pair, then in $M(A, B)$, we have $x \wedge y \in C$, for $x \in C_{A}$ and $y \in C_{B}$; and $x \vee y \in C$, for $x \in C_{A}$ and $y \in C_{B}$.

Two important examples of chopped pairs follow in which $C$ is largest and smallest possible:

Example 1. $C=(i]$ is a principal ideal of both $A$ and $B$.

We considered this special case for finite lattices in a previous paper [2]. In this case, $C_{A}=C_{B}=\varnothing$; for every $x \in M(A, B), \underline{x}=x \wedge i$; and for every $x \in C=C_{M}$, $\bar{x}=x$. The conditions of Definition 3 and Definition 4 are trivially satisfied-in fact,

$$
x \vee \bar{y}=\bar{x} \vee y=x \vee y \text { and } x \wedge \underline{y}=\underline{x} \wedge y=x \wedge y \wedge i .
$$

Example 2. $C=\{0, i\}$.

In this case, again, the conditions of Definition 3 are trivially satisfied - in fact,

$$
x \vee \bar{y}=\bar{x} \vee y=i \text { and } x \wedge \underline{y}=\underline{x} \wedge y=0 .
$$

In these two examples, the conditions of Definition 3 and Definition 4 hold in a much stronger form.

We name the first example:

Definition 5. A chopped pair of lattices, $A$ and $B$, is called full if $C=(i]_{A}=(i]_{B}$.

\section{Finitely Generated ideals}

In this section, we shall investigate conditions under which $M(A, B)$ satisfies Condition (FG). The following two lemmas are easy to verify, but they are crucial to our investigations. First some definitions. 
Definition 6. Let $A, B$ be a chopped pair, $C=A \cap B$. Let $a \in A-C$ and $b \in B-C$. We define the elements:

$$
\begin{aligned}
& a_{0}=a, \\
& b_{0}=b \text {, } \\
& b_{1}=b_{0} \vee \overline{a_{0} \wedge i} \quad \text { (formed in } B \text { ), } \\
& a_{1}=a_{0} \vee \overline{b_{1} \wedge i} \quad \text { (formed in } A \text { ), } \\
& \left.b_{2}=b_{1} \vee \overline{a_{1} \wedge i}\left(=b \vee \overline{a_{1} \wedge i}\right) \quad \text { (formed in } B\right) \text {, } \\
& a_{2}=a_{1} \vee \overline{b_{1} \wedge i}\left(=a \vee \overline{b_{1} \wedge i}\right) \quad \text { (formed in } A \text { ), } \\
& \text {... } \\
& \left.b_{n+1}=b_{n} \vee \overline{a_{n} \wedge i}\left(=b \vee \overline{a_{n} \wedge i}\right) \quad \text { (formed in } B\right) \text {, } \\
& \left.a_{n+1}=a_{n} \vee \overline{b_{n+1} \wedge i}\left(=a \vee \overline{b_{n+1} \wedge i}\right) \quad \text { (formed in } A\right) \text {, }
\end{aligned}
$$

See Figure 1-the white filled elements are in $A$ (and maybe in $C$ ); the shaded elements are in $B$ (and maybe in $C$ ), and the black filled elements are in $C$.

Lemma 5. Let $A$ and $B$ be a sharp chopped pair. Then in $M(A, B)$, the following inequalities hold:

$$
\begin{aligned}
& a=a_{0} \leq a_{1} \leq a_{2} \leq \ldots \quad(\text { in } A), \\
& b=b_{0} \leq b_{1} \leq b_{2} \leq \ldots \quad(\text { in } B),
\end{aligned}
$$

and

$$
\left.\overline{a_{0} \wedge i} \leq \overline{b_{1} \wedge i} \leq \overline{a_{1} \wedge i} \leq \overline{b_{2} \wedge i} \leq \overline{a_{2} \wedge i} \leq \ldots \text { (in } C\right) .
$$

If, for some $n, a_{n}=a_{n+1}$, then (1) terminates at $n$, and (2) terminates at $n+1$; and symmetrically, for (2). If (3) does not terminate, neither do (1) and (2).

So either all three sequences terminate or none terminate.

Proof. Let $a_{n}=a_{n+1}$; then $\overline{a_{n} \wedge i}=\overline{a_{n+1} \wedge i}$. Therefore,

$$
b_{n+2}=b \vee \overline{a_{n+1} \wedge i}=b \vee \overline{a_{n} \wedge i}=b_{n+1} ;
$$

and so $\overline{b_{n+1} \wedge i}=\overline{b_{n+2} \wedge i}$. By the definition of $a_{n+1}$ and $a_{n+2}$, it follows that $a_{n+1}=a_{n+2}$. Hence, $\overline{a_{n+1} \wedge i}=\overline{a_{n+2} \wedge i}$, so $b_{n+2}=b_{n+3}$. It is now clear that

$$
a_{n}=a_{n+1}=a_{n+2}=\ldots,
$$

and

Finally,

$$
b_{n+1}=b_{n+2}=\ldots
$$

$$
\begin{aligned}
& \quad \overline{a_{n} \wedge i} \leq \overline{b_{n+1} \wedge i} \leq \overline{a_{n+1} \wedge i} \leq \overline{b_{n+2} \wedge i}, \\
& \overline{a_{n} \wedge i}=\overline{a_{n+1} \wedge i} \text { and } \overline{b_{n+1} \wedge i}=\overline{b_{n+2} \wedge i} ; \text { therefore, }
\end{aligned}
$$

$$
\overline{a_{n} \wedge i}=\overline{b_{n+1} \wedge i}=\overline{a_{n+1} \wedge i}=\overline{b_{n+2} \wedge i}, \ldots,
$$

so sequence (3) also terminates. Conversely, if sequence (3) terminates, then sequences (1) and (2) terminate by the definitions of $a_{n+1}$ and $b_{n+1}$ in Definition 6 . 
Lemma 6. Let $A$ and $B$ be a sharp chopped pair; let $a \in A-C, b \in B-C$. The ideal $(a, b]$ of $M(A, B)$ generated by $\{a, b\}$ can be described as follows:

$$
(a, b]=\bigcup\left(\left(a_{n}\right]_{A} \mid n<\omega\right) \cup \bigcup\left(\left(b_{n}\right]_{B} \mid n<\omega\right) .
$$

This is not a finitely generated ideal if, and only if, none of the sequences of Lemma 5 terminate. If $(a, b]$ is a finitely generated ideal, then $(a, b]=\left(a_{n}\right] \cup\left(b_{n}\right]$ for some $n<\omega$.

Proof. Let $R=\bigcup\left(\left(a_{n}\right]_{A} \mid n<\omega\right) \cup \bigcup\left(\left(b_{n}\right]_{B} \mid n<\omega\right)$. If we know that $R$ is an ideal of $M(A, B)$, then it is straightforward to verify that $R$ is the ideal of $M(A, B)$ generated by $\{a, b\}$, and the rest follows from Lemma 5 .

So we verify that $R$ is an ideal of $M(A, B)$.

Firstly, let $x \in R$ and $y \leq x$ in $M(A, B)$. Without loss of generality we can assume that $x \leq a_{n}$ for some $n$ and $y \leq x$. If $y \in A$, then $y \leq a_{n}$; therefore $y \leq a_{n}$ in $A$, and so $y \in R$. If $y \in B$, then $\bar{y} \leq a_{n}$, and so $\bar{y} \leq a_{n} \wedge i \leq b_{n}$. This implies that $y \leq b_{n}$ in $B$, therefore $y \in R$; completing the proof of $y \in R$.

Secondly, let $x, y \in R$, and let $x$ and $y$ have a common upper bound $z$ in $M(A, B)$. Without loss of generality we can assume that $z \in A$. We want to show that $x \vee y \in R$. We shall distinguish several cases.

Case 1. $x, y \in A$.

Case 1.1. $x \leq a_{n}$ and $y \leq a_{m}$ for some $n$ and $m$. In this case, as in all the subsequent cases, we can assume without loss of generality that $n=m$. Then $x \vee y \leq a_{n}$, so $x \vee y \in R$.

Case 1.2. $x \leq a_{n}$ and $y \leq b_{n}$. Since $y \in A$ and $b_{n} \in B$, the condition $y \leq b_{n}$ implies that $y \leq i$. Hence, $y \leq b_{n} \wedge i \leq a_{n}$, and so $x \vee y \leq a_{n}$, yielding $x \vee y \in R$.

Case 1.3. $x \leq b_{n}$ and $y \leq a_{n}$. Proceed as in Case 1.2.

Case 1.4. $x \leq b_{n}$ and $y \leq b_{n}$. As in Case 1.2, we can verify that $x \leq a_{n}$ and $y \leq a_{n}$, so Case 1.1 completes this case.

Case 2. $x \in A, y \in B$. Observe that $y \leq i$ since $y \leq z, y \in B$ and $z \in A$.

Case 2.1. $x \leq a_{n}$ and $y \leq a_{n}$. So $x \vee y=x \vee \bar{y} \leq a_{n}$, hence $x \vee y \in R$.

Case 2.2. $x \leq a_{n}$ and $y \leq b_{n}$. Since $y \leq i$, it follows that $y \leq b_{n} \wedge i$, so $y \leq a_{n}$; hence $x \vee y \leq a_{n}$, yielding $x \vee y \in R$.

Case 2.3. $x \leq b_{n}$ and $y \leq a_{n}$. Proceed as in Case 2.2.

Case 2.4. $x \leq b_{n}$ and $y \leq b_{n}$. Then as in Case 2.2, $x \leq a_{n}$ and $y \leq a_{n}$, so we can proceed as in Case 1 .

Case 3. $x \in B, y \in A$. This is symmetric to Case 2 .

Case 4. $x, y \in B$.

Case 4.1. $x \leq a_{n}$ and $y \leq a_{n}$. Using the argument of Case 2.2, we obtain that $x \leq b_{n+1}$ and $y \leq b_{n+1}$, which is symmetric to Case 1.1. Hence $x \vee y \in R$.

Case 4.2. $x \leq a_{n}$ and $y \leq b_{n}$. Again, $x \in B$ and $x \leq a_{n}$ imply that $x \leq b_{n+1}$, which is symmetric to Case 1.1 .

Case 4.3. $x \leq b_{n}$ and $y \leq a_{n}$. Proceed as in Case 4.2.

Case 4.4. $x \leq b_{n}$ and $y \leq b_{n}$. This is symmetric to Case 1.1.

Observe that this lemma fully describes all finitely generated ideals, since a finitely generated ideal of $M(A, B)$ is obviously one- or two-generated.

Now we prove:

Theorem 2. Let $A$ and $B$ be form a sharp chopped pair, and let $C=A \cap B$. Let us assume that $C$ satisfies the Ascending Chain Condition. Then $M(A, B)$ satisfies 
condition $(\mathrm{FG})$, and $\operatorname{Id}_{\mathrm{fg}} M(A, B)$ is a congruence-preserving extension of $M(A, B)$ (in fact, a congruence-preserving $\{0\}$-extension).

Proof. If $C$ satisfies the Ascending Chain Condition, then sequence (3) of Lemma 5 must terminate. By Lemma 5, the sequences (1) and (2) terminate, and so the statement of the Theorem follows from Lemma 6 .

Finally, the statement concerning congruence-preserving extension follows from Lemma 4.

For full chopped pairs, Definition 6, Lemma 5, and Lemma 6 take on a much simpler form:

Definition 7. Let $A, B$ be a full chopped pair, $C=A \cap B$. Let $a \in A-C$ and $b \in B-C$. Then we define the elements:

$$
\begin{aligned}
a_{0} & =a, \\
b_{0} & =b, \\
b_{1} & =b_{0} \vee\left(a_{0} \wedge i\right), \\
a_{1} & =a_{0} \vee\left(b_{1} \wedge i\right), \\
b_{2} & =b_{1} \vee\left(a_{1} \wedge i\right)\left(=b \vee\left(a_{1} \wedge i\right)\right), \\
a_{2} & =a_{1} \vee\left(b_{1} \wedge i\right)\left(=a \vee\left(b_{1} \wedge i\right)\right), \\
\ldots & \\
b_{n+1} & =b_{n} \vee\left(a_{n} \wedge i\right)\left(=b \vee\left(a_{n} \wedge i\right)\right), \\
a_{n+1} & =a_{n} \vee\left(b_{n+1} \wedge i\right)\left(=a \vee\left(b_{n+1} \wedge i\right)\right), \\
\ldots &
\end{aligned}
$$

See Figure 2-the white filled elements are in $A$ (and maybe in $C$ ); the shaded elements are in $B$ (and maybe in $C$ ), and the black filled elements are in $C$.

Lemma 7. Let $A$ and $B$ be a full chopped pair. Then in $M(A, B)$, the following inequalities hold:

$$
\begin{gathered}
a=a_{0} \leq a_{1} \leq a_{2} \leq \ldots(\text { in } A), \\
b=b_{0} \leq b_{1} \leq b_{2} \leq \ldots(\text { in } B)
\end{gathered}
$$

and

$$
\left.a_{0} \wedge i \leq b_{1} \wedge i \leq a_{1} \wedge i \leq b_{2} \wedge i \leq a_{2} \wedge i \leq \ldots \text { (in } C\right) .
$$

If, for some $n, a_{n}=a_{n+1}$, then (4) terminates at $n$, and (5) terminates at $n+1$; and symmetrically, for (5). If (6) does not terminate, neither do (4) and (5).

The proof of this lemma is a simplified version of the proof of Lemma 5 . Lemma 6 remains valid for full chopped pairs; in this case, the sequences $a_{n}$ and $b_{n}$ will be the ones defined in Definition 7.

\section{Modular lattices}

By inspecting Figure 1, we can see that if $A$ and $B$ are modular, then a lot of elements must collapse. In fact, we have the following result: 
Theorem 3. Let $A$ and $B$ form a sharp chopped pair. Let us assume that both $A$ and $B$ are modular. Then $M(A, B)$ satisfies condition $(\mathrm{FG})$, and $\operatorname{Id}_{\mathrm{fg}} M(A, B)$ is a congruence-preserving extension of $M(A, B)$ (in fact, a congruence-preserving $\{0\}$-extension).

Proof. Let $A$ and $B$ be modular. The equations (see Figure 1)

$$
\begin{aligned}
& a_{0} \wedge \overline{b_{1} \wedge i}=a_{0} \wedge\left(a_{1} \wedge i\right)=a_{0} \wedge i, \\
& a_{0} \vee \overline{b_{1} \wedge i}=a_{0} \vee\left(a_{1} \wedge i\right)=a_{1}
\end{aligned}
$$

hold in $M(A, B)$. By the modularity of $A$, the two equations imply that $\overline{b_{1} \wedge i}=$ $a_{1} \wedge i$. So

$$
\overline{a_{1} \wedge i}=\overline{\overline{b_{1} \wedge i}}=\overline{b_{1} \wedge i} .
$$

By the modularity of $B$, a similar argument yields that $\overline{b_{2} \wedge i}=\overline{a_{1} \wedge i}$, and so on. So the sequence (3) has only one or two members; it terminates. By Lemma 5 , the sequences (1) and (2) terminate. So the statement of the Theorem follows from Lemma 6.

Finally, the statement concerning congruence-preserving extension follows from Lemma 4.

We can prove a stronger statement for full chopped pairs.

Lemma 8. Let $A, B$ be a full chopped pair. If $A$ is a modular lattice, then

$$
(a, b]=\left(a_{1}\right] \cup\left(b_{1}\right] .
$$

Proof. As in Theorem 3, the modularity of $A$ implies that $b_{1} \wedge i=a_{1} \wedge i$. Hence $b_{2}=b_{1} \vee\left(a_{1} \wedge i\right)=b_{1} \vee\left(b_{1} \wedge i\right)=b_{1}$, and $a_{2}=a_{1} \vee\left(b_{2} \wedge i\right)=a_{1} \vee\left(b_{1} \wedge i\right)=$ $a_{1} \vee\left(a_{1} \wedge i\right)=a_{1}$. So the statement of the Lemma follows from Lemma 6 .

So now we can conclude a stronger form of Theorem 3 for full chopped pairs:

Theorem 4. Let $A, B$ be a full chopped pair. If $A$ is a modular lattice, then $M(A, B)$ satisfies condition $(\mathrm{FG})$.

\section{CONGRUENCE-PRESERVING EXTENSIONS}

In [2] we raised the following question:

Problem . Is it true that every lattice with more than one element has a proper congruence-preserving extension?

We proved in [2] that in the finite case this is true. This result is generalized by the following theorem:

Theorem 5. Let $L$ be a lattice with zero, 0 . If there exists an element $\alpha>0$ in $L$ such that the interval $[0, \alpha]$ is distributive, then $L$ has a proper congruence-preserving extension $K$.

Proof. To prove this result, we need a construction due to the second author. Let $M_{3}$ denote the five-element modular nondistributive lattice on the set $\{0, a, b, c, 1\}$, and let $D$ be a bounded distributive lattice. Let

$$
M_{3}[D]=\left\{\langle x, y, z\rangle \in D^{3} \mid x \wedge y=x \wedge z=y \wedge z\right\} .
$$

Then $M_{3}[D]$ is a modular lattice; it contains $M_{3}$ as a $\{0,1\}$-sublattice (on the set $\{\langle 0,0,0\rangle,\langle 1,0,0\rangle,\langle 0,1,0\rangle,\langle 0,0,1\rangle,\langle 1,1,1\rangle\})$, and each prime interval of this $M_{3}$ 
contains (in $M_{3}[D]$ ) a copy of $D$; for instance, the interval $[\langle 0,0,0\rangle,\langle 1,0,0\rangle]$ can be described as $\{\langle d, 0,0\rangle \mid d \in D\}$. If we identify $D$ with $\{\langle d, 0,0\rangle \mid d \in D\}$, we find that the lattice $M_{3}[D]$ is a congruence-preserving $\{0\}$-extension of $D$.

Now let $D=[0, \alpha]$, and let $A=M_{3}[D]$. Then $A$ has a spanning $M_{3}$; let $i=\langle a, 0,0\rangle$. Let $B=L$, and define $i=\alpha$ in $B$. Then $A \cap B=(i]$, and $A$, $B$ form a full chopped pair in which $A$ is modular. So we can form the chopped lattice $M(A, B)$. Obviously, $M(A, B)$ is a proper congruence-preserving $\{0\}$-extension of $L$. By Theorem 4, (FG) holds for $M(A, B)$. Therefore, by Lemma 4, $\operatorname{Id}_{\mathrm{fg}} M(A, B)$ is a congruence-preserving $\{0\}$-extension of $M(A, B)$. We conclude that $\operatorname{Id}_{\mathrm{fg}} M(A, B)$ is a proper congruence-preserving $\{0\}$-extension of $L$.

The following result is a generalization of Theorem 5 .

Theorem 6. Let $L$ be a lattice. If there exist a nontrivial distributive interval in $L$, then $L$ has a proper congruence-preserving extension $K$.

Proof. Let $[\alpha, \beta]$ be a nontrivial distributive interval in $L$. Let us form the lattice $B=[\alpha)$ in $L$. Obviously, $B$ satisfies the conditions of Theorem 5 ; therefore, $B$ has a congruence-preserving $\{0\}$-extension $K_{1}$. Clearly, $B$ is an ideal of $K_{1}$ and a dual ideal of $L$; hence we can glue $L$ and $K_{1}$ over $B$; let $K$ be the resulting lattice.

Let $\Theta$ be a congruence relation on $L$. Let $\Theta_{B}$ be the restriction of $\Theta$ to $B$. Since $K_{1}$ is a congruence-preserving extension of $B$, there is a unique extension $\Phi$ of $\Theta_{B}$ to $K_{1}$. It is easy to see that $\bar{\Theta}=\Theta \cup \Phi$ is the unique extension of $\Theta$ to $K$. Hence $K$ is a congruence-preserving extension of $L$. Obviously, it is a proper extension.

\section{A MOdUlar EXAMPLE}

It is easy to give examples of classes of lattices that have proper congruence-preserving extensions that have nothing to do with distributivity. For instance, every simple lattice with more than one element has a proper simple extension; this is obviously a proper congruence-preserving extension.

In this section we outline a modular example with no proper distributive sublattice.

Let $C$ be a continuous geometry with zero, 0 , and unit, 1 . Then $C$ has the following properties:

1. For $a<b$, the interval $[a, b]$ is isomorphic to $C$.

2. $C$ is a simple lattice.

Let $I$ be a nonprincipal ideal of $C$ and $F$ a nonprincipal dual ideal of $C$ satisfying $I \cap F=\varnothing$. Let $L$ be the sublattice $I \cup F$. The congruence lattice of $L$ is the three element chain.

We choose in $C$ a spanning $M_{3}=\{0<a, b, c<1\}$. The interval $[0, a]$ is isomorphic to $C$. Therefore, we find in $[0, a]$ a copy $I_{a}$ of $I$ and a copy $F_{a}$ of $F$. The projectivities in the spanning $M_{3}$ define the ideals and dual ideals, $I_{b}, I_{c}, F_{b}$, $F_{c}$ in the intervals $[0, b]$ and $[0, c]$. Similarly, we obtain the ideal $I_{a}^{u}$ and dual ideal $F_{a}^{u}$ in $[a, 1], I_{b}^{u}$ and $F_{b}^{u}$ in $[b, 1], I_{c}^{u}$ and $F_{c}^{u}$ in $[c, 1]$.

Let $I$ be the ideal of $C$ generated by the three "small" ideals, $I_{a}, I_{b}, I_{c}$. Similarly, the three dual ideals $F_{a}^{u}, F_{b}^{u}, F_{c}^{u}$ generate a dual ideal $F$. We consider the sublattice

$$
K=I \cup F \cup F_{a} \cup F_{b} \cup F_{c} \cup I_{a}^{u} \cup I_{b}^{u} \cup I_{c}^{u} .
$$

It is easy to see that $K$ is a sublattice of $C$, and it is a congruence-preserving extension of the sublattice $L \subseteq[0, a]$. 


\section{REFERENCES}

[1] G. Grätzer, General Lattice Theory, Academic Press, New York, N. Y., Birkhäuser Verlag, Basel, Akademie Verlag, Berlin, 1978.

[2] G. Grätzer and E. T. Schmidt, The Strong Independence Theorem for automorphism groups and congruence lattices of finite lattices. Manuscript.

Department of Mathematics, University of Manitoba, Winnipeg, Man. R3T 2N2, CANADA

E-mail address: George_Gratzer@umanitoba.ca

Department of Mathematics, Transport Engineering Faculty, Technical University OF BudAPEST, MÚegyetem RKP. 9, 1111 BudAPEST, Hungary

E-mail address: h1175sch@ella.hu 